Research Article

\title{
Vibration-Based, Nondestructive Methodology for Detecting Multiple Cracks in Bending-Torsion Coupled Laminated Composite Beams
}

\author{
Stephen R. Borneman ${ }^{1}$ and Seyed M. Hashemi ${ }^{2}{ }^{2}$ \\ ${ }^{1}$ Babcock Canada, 75 Albert Street, Suite 800, Ottawa, ON, Canada K1P 5E7 \\ ${ }^{2}$ Department of Aertospace Engineering, Ryerson University, 350 Victoria Street, Toronto, ON, Canada M5B 2 K3
}

Correspondence should be addressed to Seyed M. Hashemi; smhashem@ryerson.ca

Received 31 May 2018; Accepted 25 July 2018; Published 26 August 2018

Academic Editor: Daniele Baraldi

Copyright (c) 2018 Stephen R. Borneman and Seyed M. Hashemi. This is an open access article distributed under the Creative Commons Attribution License, which permits unrestricted use, distribution, and reproduction in any medium, provided the original work is properly cited.

\begin{abstract}
Damage to composite structures occurs from impact, fatigue, or over stress and can be critical in the safe operation of wings or any structural member. This paper presents a method for detection of multiple cracks present in laminated composite bending-torsion coupled cantilevered beams using natural frequency data, a type of Nondestructive testing (NDT). This methodology relies on both experimentally collected natural frequencies and frequencies calculated using a mathematical model. Precise natural frequencies are calculated using a new dynamic finite cracked element (DFCE) formulated within and based on dynamic trigonometric shape functions. An algorithm is devised based on the Adam-Cawley criterion and extended to laminated composites with multiple cracks. This method has shown exceptional convergence on the size and location of cracks using a number of modes of free vibration with and without error in measured frequencies.
\end{abstract}

\section{Introduction}

A wealth of information is bundled up in a sequence of numbers that define the way in which beams deform in time. Lightweight structures with complex anisotropic behavior are trending in industry because of their low weight to stiffness/strength ratio. Additionally, the stiffness of these materials can be tailored through ply orientation and stacking sequence to achieve unique modal responses tailored for specific applications. Analytical methods for evaluating the vibration of laminated composite structures have progressed rapidly in recent years incorporating more complex behaviors to facilitate a more accurate evaluation of dynamics, structural integrity, and fatigue. Natural frequencies are numbers that describe the cyclic time required for a flexible structure to take forms in correspondingly naturally deformed shapes or modes. Knowledge of these frequencies can be used to avoid stability issues in design of buildings, bridges, and any other structures where natural frequencies are critical. Wings are common cantilevered beam-like structures, where the knowledge of crack initiation or propagation is critical in flight safety. Currently, the structural integrity of aircraft wings is tested when aircraft are grounded, generally with X-ray scanning equipment. Although this process can be time consuming, the safety of passengers and operators is paramount and essential in preventing catastrophic failure of commercial or military aircraft. Wings consist of a unique set of natural frequencies that are fundamentally based on mass, stiffness, and geometry. If the mass and geometry of a beam is constant, any changes in natural frequencies can be attributed to stiffness. When a crack is introduced in a beam, an instantaneous drop in stiffness consequently results in a change in the characteristic frequencies. Analyzing and tracking these changes by implementing the correct methodology can provide significant information about a structure and its integrity.

This paper involves the study of how natural frequencies can be used to find cracks of various sizes and locations in laminated composite Euler-Bernoulli and St. Venant beams nondestructively, a type of structural health monitoring. A number of authors in the past have shown how altered mode 
shapes can be visually inspected in an attempt to indicate possible defects in beams; however, often the size and location of the crack remains ambiguous and difficult to determine with confidence. Correspondingly, this becomes more difficult when errors in measured data are accounted for, or if the size of the crack is small, visual detection of cracks using modes can be very difficult, particularly when modes often contain noise. This research provides a definitive method to find defects in complex coupled structures such as laminated composite Euler-Bernoulli and St. Venant cantilever beams by simply using frequency data. Collecting frequency data experimentally from a structure with an unknown defect and subsequently comparing these data with various natural frequencies obtained from a model with a known defect can provide detection of the size and location of structural defects in a wing if these data are managed by an appropriate algorithm. A crack can initiate from over stress, impact, or fatigue, subsequently generating reduction in stiffness and altering fundamental vibrations.

Cawley and Adams [1] first proposed a method for determining the location of cracks in a cylindrical geometry composed of homogeneous material using frequency data. In contrast, there exist crack detection approaches that rely solely on visual inspection of discontinuous natural modes; however, visual changes in mode shapes can be difficult to isolate crack locations and corresponding sizes, especially when considering complex coupled responses inherent in composites. A good review on damage identification methods is done by Doebling et al. [2] who compared a number of approaches including frequency-based crack detection, one of which is the Adam-Cawley criterion [3], which was developed to determine the size and location of cracks in homogeneous materials, and subsequently provided excellent results. The criterion is much less accurate when used to detect cracks in composite material where modes of free vibration are naturally coupled. As a result, Wang [4] devised a way to overcome this problem by resequencing the modes by ordering the frequencies by the magnitude of the difference between intact and crack frequencies from largest to smallest change. Wang's enhancement to the Adam-Cawley criterion has shown to produce excellent detection of the size and location of cracks in bending-torsion coupled composite material. This enhanced criterion can be described as a damage index that involves the ratio of the change in experimental frequencies with the change in frequencies calculated by model prediction. These changes in both experimental and calculated frequencies are evaluated by taking the difference of these frequencies from their respective intact frequency values. This forms a basis for the damage index which is then normalized such that the size and location of a crack are indicated by unity.

A multiple crack detection strategy is devised herein based on this criterion by implementing dual damage indices capable of confidently detecting two or more cracks for size and location. The introduction of this second indicator ultimately provides a measure of the primary indicator, simultaneously exposing both cracks. Accordingly, multiple cracks are evaluated for both size and location using sequential order of modes and implementation of dual damage indices. Correspondingly, it is important to have an accurate model of a defective composite beam for the crack detection to be successful. This is accomplished with dynamic finite elements (DFEs) combined with the appropriate local compliance model of the cracks in a coupled bending-torsion composite beam, thus establishing a dynamic finite cracked element (DFCE). DFE has been validated by comparison of both the finite element method (FEM) and the exact solution of the natural frequencies of various beams. Although this paper presents a new DFCE for damaged beams, it is envisioned that the methodology could also be extended to twodimensional structural elements based on a new DFE recently formulated for thin rectangular plates [5]. A laminated composite DFE stiffness matrix is similar to the exact DSM (dynamic stiffness matrix) [6], having a frequency-dependent stiffness matrix, and is solved to extract the natural frequencies using the Wittrick-William algorithm [7].

With these enhanced dynamic elements and the methodology introduced in this paper, a highly robust detection algorithm is capable of detecting multiple through thickness cracks in a composite beam.

\section{Dynamic Finite Cracked Element (DFCE)}

Crack detection using natural frequencies relies considerably on the accuracy of the representative of the beam. DFEs have been shown in Reference [8] to provide highly accurate natural frequencies for various beam geometries, for example, uniform and nonlinear geometries and materials. For this reason, the following formulation of a DFCE is created and achieved using two DFEs adjoined with a local compliance (Figure 1), where $y_{c}$ represents the length of the beam from the fixed boundary along the span to the crack and $L$ denotes the length of the beam. Also, $b$ and $t$ denote the width and thickness of the beam, respectively.

The governing differentials governing the motion of a coupled bend-twist composite beam based on the Euler-Bernoulli bending and St. Venant torsion are given in the following:

$$
\begin{aligned}
& E I \frac{\partial^{4} h}{\partial y^{4}}+K \frac{\partial^{3} \psi}{\partial y^{3}}+m \frac{\partial^{2} h}{\partial t^{2}}=0 \\
& G J \frac{\partial^{2} h}{\partial y^{2}}+K \frac{\partial^{3} h}{\partial y^{3}}+I_{\alpha} \frac{\partial^{2} \psi}{\partial t^{2}}=0
\end{aligned}
$$

where $E I, G J$, and $K$ are the bending, twisting, and coupled bending-twisting rigidity of the laminated composite beam, respectively, and defined in Appendix A, based on reduced stiffness coefficients. $m$ denotes the mass per unit length, and $I_{\alpha}$ denotes mass moment of inertia per unit length of the composite beam. These equations of motion are strictly valid for symmetric stacking and unidirectional unbalanced stacking of laminate plies. The following variables for bending and twisting displacements are defined based on the assumption of harmonic motion:

$$
\begin{aligned}
& h(y, t)=H(y) \exp ^{i \omega t}, \\
& \psi(y, t)=\Psi(y) \exp ^{i \omega t} .
\end{aligned}
$$




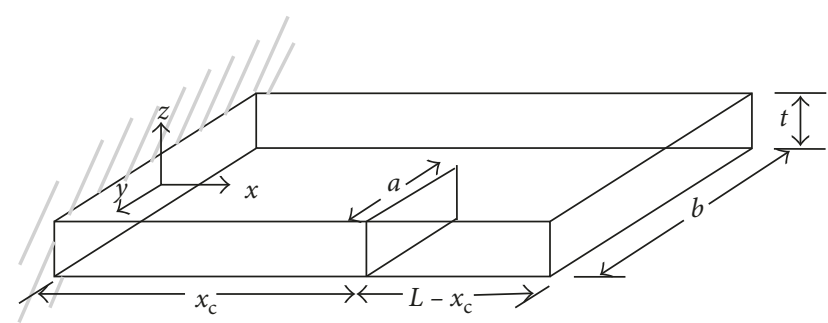

FIgURE 1: Geometry of a laminated cracked composite beam.

The Galerkin weighted residual method is used, appropriate integration by parts is applied, and the continuity requirements on the field variables $H$ and $\Psi$ are relaxed to $C_{1}$ and $C_{0}$, respectively, so that the integral weak form can then be obtained. The resulting weak integrals are then split over two domains $\left(0 \leq y \leq y_{\mathrm{c}}\right.$ and $\left.y_{\mathrm{c}} \leq y \leq L\right)$, each representing one of the intact beam segments adjoined at the crack location. The weak form of flexural motion is then

$$
\begin{aligned}
W_{\mathrm{f}}= & \int_{0}^{y_{\mathrm{c}}}\left[E I(y) H_{1, y y} \delta H_{1, y y}+K(y) \Psi_{1, y} \delta H_{1, y y}\right. \\
& \left.-m(y) \omega^{2} H_{1} \delta H_{1}\right] d y+\int_{y_{\mathrm{c}}}^{L}\left[E I(y) H_{2, y y} \delta H_{2, y y}\right. \\
& \left.+K(y) \Psi_{2, y} \delta H_{2, y y}-m(y) \omega^{2} H_{2} \delta H_{2}\right] d y \\
& +\left[\left(E I(y) H_{1, y y}+K(y) \Psi_{1, y}\right)_{y} \delta H_{1}\right]_{0}^{y_{c}} \\
& -\left[\left(E I(y) H_{1, y y}+K(y) \Psi_{1, y}\right) \delta H_{1, y}\right]_{0}^{y_{c}} \\
& +\left[\left(E I(y) H_{2, y y}+K(y) \Psi_{2, y}\right) \delta H_{2}\right]_{y_{c}}^{L} \\
& -\left[\left(E I(y) H_{2, y y}+K(y) \Psi_{2, y}\right) \delta H_{2, y}\right]_{y_{c}}^{L} .
\end{aligned}
$$

And twisting motion is

$$
\begin{aligned}
W_{\mathrm{t}}= & \int_{0}^{y_{\mathrm{c}}}\left[-G J(y) \Psi_{1, y} \delta \Psi_{1, y}-K(y) H_{1, y y} \delta \Psi_{1}\right. \\
& \left.+I_{\alpha}(y) \omega^{2} \Psi_{1} \delta \Psi_{1}\right] d y+\int_{0}^{y_{c}}\left[-G J(y) \Psi_{2, y} \delta \Psi_{2, y}\right. \\
& \left.-K(y) H_{2, y y} \delta \Psi_{2}+I_{\alpha}(y) \omega^{2} \Psi_{2} \delta \Psi_{2}\right] d y \\
& +\left[\left(G J(y) \Psi_{1, y}+K(y) H_{1, y y}\right) \delta \Psi_{1}\right]_{0}^{y_{c}} \\
& +\left[\left(G J(y) \Psi_{2, y}+K(y) H_{2, y y}\right) \delta \Psi_{2}\right]_{0}^{y_{c}},
\end{aligned}
$$

which also satisfies the principle of virtual work $\left(W_{\mathrm{EXT}}=0\right.$, for free vibrations):

$$
W_{\mathrm{f}}+W_{\mathrm{t}}=\text { total internal virtual work. }
$$

Boundary conditions associated with a clamped-free cantilever beam are such that

$$
\begin{aligned}
@ y=0, \quad H_{1}(0) & =\theta_{1}(0)=\Psi(0)=0, \\
\delta H_{1} & =\delta \theta_{1}=\delta \Psi_{1}=0, \text { and } \\
@ y=L, \quad S_{h 2}(L) & =M_{2}(L)=T_{2}(L)=0,
\end{aligned}
$$

where resulting shear force, $S_{h}(y)$, bending moment, $M(y)$, and torsional torque, $T(y)$, are

$$
\begin{aligned}
& S_{h}(y)=\frac{d}{d y}\left(E I(y) \frac{d^{2} H}{d y^{2}}+K(y) \frac{d \Psi}{d y}\right), \\
& M(y)=-E I(y) \frac{d^{2} H}{d y^{2}}-K(y) \frac{d \Psi}{d y}, \\
& T(y)=G J(y) \frac{d \Psi}{d y}+K(y) \frac{d^{2} H}{d y^{2}} .
\end{aligned}
$$

The nonzero boundary conditions (Figure 2) generated at the crack can be written in the following matrix form:

$$
\begin{aligned}
W_{\mathrm{f}}^{\mathrm{BC}} & =\left[\begin{array}{cccc}
S_{h 1}(y) & 0 & 0 & 0 \\
0 & M_{1}(y) & 0 & 0 \\
0 & 0 & S_{h 2}(y) & 0 \\
0 & 0 & 0 & M_{2}(y)
\end{array}\right] \text { and } \\
W_{\mathrm{t}}^{\mathrm{BC}} & =\left[\begin{array}{cc}
T_{1}(y) & 0 \\
0 & T_{2}(y)
\end{array}\right] .
\end{aligned}
$$

A local flexibility at the crack location is developed by combining two intact beam members with a spring, representing the stiffness at the crack [4]. The natural frequencies can then be calculated for this damaged composite beam structure. Forces and moments are continuous across the crack, whereas displacements are discontinuous and given by the following expression:

$$
\left\{\begin{array}{c}
H_{2} \\
H_{2, y} \\
\Psi_{2, y}
\end{array}\right\}=\left[\begin{array}{ccc}
c_{22} & 0 & c_{26} \\
0 & c_{44} & 0 \\
c_{26} & 0 & c_{66}
\end{array}\right]\left\{\begin{array}{c}
S_{h} \\
M \\
T
\end{array}\right\}+\left\{\begin{array}{c}
H_{1} \\
H_{1, y} \\
\Psi_{1}
\end{array}\right\},
$$

where $c_{22}, c_{44}, c_{26}$, and $c_{66}$ are the components of the local flexibility at the crack location and are based on the stress intensity factors and correction functions corresponding to a particular mode of the crack. These components are defined by the following equation:

$$
\begin{aligned}
c_{i j}= & \frac{\partial^{2}}{\partial P_{i} \partial P_{j}} \int_{-t / 2}^{t / 2} \int_{0}^{a}\left\{D_{1}\left(K_{\mathrm{I} 1}+K_{\mathrm{I} 4}+K_{\mathrm{II}}\right)^{2}+D_{2} K_{\mathrm{II} 3}^{2}\right. \\
& \left.+D_{12}\left(K_{\mathrm{I} 1}+K_{\mathrm{I} 4}+K_{\mathrm{I} 5}\right) K_{\mathrm{II} 3}+D_{3}\left(K_{\mathrm{III} 3}+K_{\mathrm{III} 6}\right)^{2}\right\} d \alpha d z .
\end{aligned}
$$

Fundamentally, three distinct modes of a crack are illustrated in Figure 3.

$K_{\mathrm{I}}, K_{\mathrm{II}}$, and $K_{\mathrm{III}}$ are the stress intensity factors, respectively, for each crack mode. Coefficients $D_{1}, D_{2}, D_{12}$, and $D_{3}$ were developed by Nikpour et al. [9] to describe the strain energy release rate per unit crack width. A local flexibility is then defined for the crack location (Figure 4) using these stress intensity factors, based in part by Tada's geometric correction functions [10] (homogeneous material), and also corrected for material anisotropy by Bao et al. [11]. Accordingly, the final stiffness matrix representing the local flexibility at the crack location (Figure 4) can be derived as

$$
K_{\mathrm{c}}=\left[\begin{array}{cc}
{[C]^{-1}} & -[C]^{-1} \\
-[C]^{-1} & {[C]^{-1}}
\end{array}\right] .
$$

Integration by parts is performed second time on the system after the equations have been discretized over the 


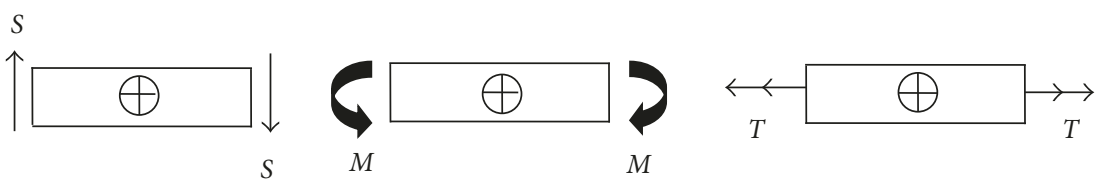

Figure 2: Sign convention, where $S$ denotes the transverse force, $M$ denotes the bending moment, and $T$ denotes the torque.

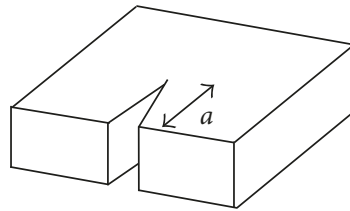

(a)

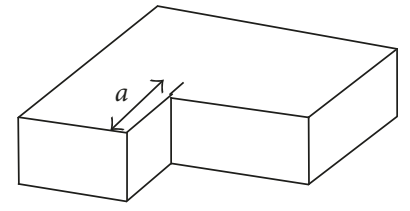

(b)

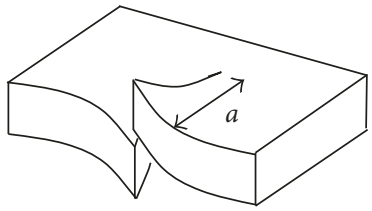

(c)

Figure 3: Three fundamental modes of a crack. (a) Mode I: opening of the crack (extension). (b) Mode II: sliding of the crack (shearing). (c) Mode III: tearing of the crack (twisting).

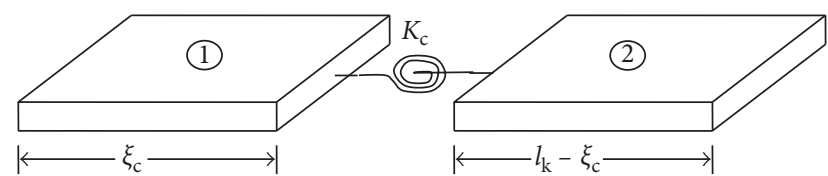

Figure 4: Cracked beam element.

length of the beam. Nondimensional bending and torsional contributions to the element's virtual work, $W_{\mathrm{f}}^{k}$ and $W_{\mathrm{t}}^{k}$, respectively, are

$$
\begin{aligned}
& W_{\mathrm{f}}^{k}=\int_{0}^{\xi_{\mathrm{c}}} H_{1}[\underbrace{E I / l_{k}^{3} \delta H_{1, \xi \xi \xi \xi}-l_{k} m \omega^{2} \delta H_{1}}_{{ }^{*} a_{1}}] d \xi \\
& +\int_{\xi_{c}}^{1} H_{2}[\underbrace{E I / l_{k}^{3} \delta H_{2, \xi \xi \xi \xi}-l_{k} m \omega^{2} \delta H_{2}}_{{ }^{*} a_{2}}] d \xi \\
& +\frac{E I}{l_{k}^{3}}\left[\delta H_{1, \xi \xi} H_{1, \xi}-\delta H_{1, \xi \xi \xi} H_{1}\right]_{0}^{\xi_{c}} \\
& +\frac{E I}{l_{k}^{3}}\left[\delta H_{2, \xi \xi} H_{2, \xi}-\delta H_{2, \xi \xi \xi} H_{2}\right]_{0}^{\xi_{c}} \\
& +\frac{K}{l_{k}^{2}} \int_{0}^{\xi_{c}} \Psi_{1, \xi} \delta H_{1, \xi \xi} d \xi+\frac{K}{l_{k}^{2}} \int_{\xi_{c}}^{1} \Psi_{2, \xi} \delta H_{2, \xi \xi} d \xi \\
& +W_{\mathrm{f}}^{\mathrm{BC}}\left(\xi_{\mathrm{c}}\right) \text { and } \\
& W_{\mathrm{t}}^{k}=\int_{0}^{\xi_{\mathrm{c}}} \Psi_{1}[\underbrace{-G J / l_{k} \delta \Psi_{1, \xi \xi}-l_{k} I_{\alpha} \omega^{2} \delta \Psi_{1}}_{{ }^{*} b_{1}}] d \xi \\
& +\int_{\xi_{c}}^{1} \Psi_{2}[\underbrace{-G J / l_{k} \delta \Psi_{2, \xi \xi}-l_{k} I_{\alpha} \omega^{2} \delta \Psi_{2}}_{{ }^{*} b_{2}}] d \xi \\
& +\frac{G J}{l_{k}}\left[\delta \Psi_{1, \xi} \Psi_{1}\right]_{0}^{\xi_{c}}+\frac{G J}{l_{k}}\left[\delta \Psi_{2, \xi} \Psi_{2}\right]_{\xi_{c}}^{1} \\
& +\frac{K}{l_{k}^{2}} \int_{0}^{\xi_{\mathrm{c}}} H_{1, \xi \xi} \delta \Psi_{1, \xi} d \xi+\frac{K}{l_{k}^{2}} \int_{\xi_{\mathrm{c}}}^{1} H_{2, \xi \xi} \delta \Psi_{2, \xi} d \xi \\
& +W_{\mathrm{t}}^{\mathrm{BC}}\left(\xi_{\mathrm{c}}\right) \text {, }
\end{aligned}
$$

where the length of the beam is discretized to $\xi=y / l_{k}$, satisfying

$$
\sum_{k=1}^{\mathrm{NE}} W_{\mathrm{f}}^{k}+\sum_{k=1}^{\mathrm{NE}} W_{\mathrm{t}}^{k}=0 .
$$

Local virtual work expressions evaluated at the crack boundary, $W_{\mathrm{f}}^{\mathrm{BC}}\left(\xi_{\mathrm{c}}\right)$ and $W_{\mathrm{t}}^{\mathrm{BC}}\left(\xi_{\mathrm{c}}\right)$, can be replaced in the stiffness matrix with the new local flexibility matrix. DFE is a hybrid numerical method combining the accuracy of the DSM method [6] with the finite element method (FEM). Shape functions are derived such that integral expressions ${ }^{*} a_{1},{ }^{*} a_{2},{ }^{*} b_{1}$, and ${ }^{*} b_{2}$ in (9) and (10) are equal to zero. Consequently, dynamic trigonometric shape functions must be frequency dependent (Appendix B), and accordingly, the final globally stiffness matrix is also frequency dependent and given as

$$
K=K_{\mathrm{IN}, 1}+K_{\mathrm{c}}+K_{\mathrm{IN}, 2} \text {. }
$$

This final frequency-dependent stiffness matrix, denoted by $K$, represents the stiffness of a cracked beam element, where $K_{\mathrm{IN}, 1}$ and $K_{\mathrm{IN}, 2}$ are the intact beam stiffness matrices and $K_{\mathrm{c}}$ is the local crack stiffness. This results in the following nonlinear eigenvalue problem:

$$
\left[K(\omega)\{U\}_{n}\right]=\{0\} .
$$

The solution of the natural frequencies pertaining to this nonlinear eigenvalue problem can be attained by implementation of the Wittrick-William root counting algorithm [7].

\section{Multiple Crack Detection}

In this section, a frequency-based strategy to detect both multiple sizes and locations of cracks within a damaged laminated composite cantilevered beam is presented. The technique was first devised by Adams et al. [3] for revealing the presence of a single crack in homogeneous material. Naturally, by inspection of the modal response of a structure, if the natural frequencies are lower as compared to its original measurements, it can be reasonably postulated that there is a corresponding drop in stiffness potentially indicating a discontinuity along the length of the beam. 
Although changes in natural frequencies of a structure may indicate the presence of a crack, the challenge is using these data to determine how large and the location of such a local damage(s). The following methodology is based on dual damage indices, where the primary index follows the equation derived by the Cawley-Adams and the second index evaluates the accuracy of the primary index. Correspondingly, the second index simultaneously determines the locations and sizes of both cracks. In the following definitions, it is important to be consistent with the definition of each crack; therefore, crack-1 is defined as the discontinuity closest to the fixed boundary of the cantilevered beam, whereas crack- 2 is defined as the crack closest to the free end of the beam. The frequency ratio of different modes, $i$ and $j$, as a function of both crack size and location for crack- 1 and crack-2 is defined as

$$
\begin{array}{r}
e_{i j}\left(\alpha_{1}, \eta_{1}, \alpha_{2}, \eta_{2}\right)= \begin{cases}\frac{\delta \lambda_{i} / \delta \lambda_{j}}{\delta \omega_{i} / \delta \omega_{j}}-1 & \text { if } \frac{\delta \lambda_{i}}{\delta \lambda_{j}} \geq \frac{\delta \omega_{i}}{\delta \omega_{j}}, \\
\frac{\delta \omega_{i} / \delta \omega_{j}}{\delta \lambda_{i} / \delta \lambda_{j}}-1 & \text { if } \frac{\delta \omega_{i}}{\delta \omega_{j}} \geq \frac{\delta \lambda_{i}}{\delta \lambda_{j},}\end{cases} \\
\text { for }\left\{\begin{array}{l}
i=1,2,3, \ldots, n-1, \\
j=2,3, \ldots, n,
\end{array}\right.
\end{array}
$$

where $\delta \omega$ is the change in frequency between intact and damaged frequencies by model prediction and $\delta \lambda$ is the change in frequency between intact and damaged frequencies collected by experiment. The size and location of crack-1 are denoted by $\alpha_{1}$ and $\eta_{1}$, respectively, whereas the second crack by $\alpha_{2}$ and $\eta_{2}$. For each crack-2 size and location, there exists a normalized primary indicator, $E_{q r s t}$, with counters $q, r, s$, and $t$, which are equal to $10 \alpha_{1}, 10 \eta_{1}$, $10 \alpha_{2}$, and $10 \eta_{2}$, respectively.

$$
E_{\text {qrst }}\left(\alpha_{1}, \eta_{1}, \alpha_{2}, \eta_{2}\right)=\frac{\min \left(\zeta_{q r s t}\right)}{\zeta_{q r s t}\left(\alpha_{1}, \eta_{1}, \alpha_{2}, \eta_{2}\right)}, \quad 0 \leq E_{\text {qrst }} \leq 1 .
$$

For each crack-1 size and location, there exists $(s \times t)-1$ primary indices for crack-2, where

$$
\zeta_{\text {qrst }}\left(\alpha_{1}, \eta_{1}, \alpha_{2}, \eta_{2}\right)=\sum_{i=1}^{n-1} \sum_{i=i+1}^{n} e_{i j}\left(\alpha_{1}, \eta_{1}, \alpha_{2}, \eta_{2}\right) .
$$

The normalized damage index $E_{\text {qrst }}$ indicates unity when crack-2 is likely found, and less than unity for crack-2 size $\alpha_{2}$ and location $\eta_{2}$ that do not coincide with a crack. The key is to determine which primary index is indicating the correct crack-2. This is accomplished by implementing a second index to measure the relative accuracy of each $(s \times t)-1$ primary index using the following equation:

$$
F_{q r}=\frac{-1}{\ln \left(\mu_{q r}-1\right)},
$$

where

$$
\mu_{q r}=\frac{\left(\sum_{s=1}^{k} \sum_{t=1}^{l} E_{q r s t}\left(\alpha_{1}, \eta_{1}, \alpha_{2}, \eta_{2}\right)-1\right)}{(k l-1)},
$$

where $k$ is the total number of incremental locations and $l$ is the total number of incremental sizes sweeping across the beam for the second crack. The accuracy of this primary indicator is then evaluated by this normalized secondary indicator, $F_{q r}$. The secondary index provides a value of unity for the correct primary index. Subsequently, the secondary index indicates the size and location of the first crack while simultaneously indicating which primary index is specifying the correct size and location of crack-2.

Detection becomes more difficult when considering error in measured frequencies. However, by increasing the number of modes when computing the two indices more precision in the detection is achieved diminishing the effects of this error. The number of modes is increased until the prediction of both the primary and secondary indices is unchanged for the location and size of each crack (i.e., $F_{q r}$ and $E_{\text {qrst }}$ remain constant with increasing modes).

\section{Numerical Examples}

4.1. Free Vibration and Validation. Evaluation of the free vibration of a damaged Euler-Bernouilli and St. Venant torsion uniform cantilevered composite beam is demonstrated in the following example. By implementing the theory of a new dynamic finite cracked element (DFCE) based on the theoretical development in Section 2 and comparing these results with the well-established exact closed-form DSM solution, validation of a new cracked element is confirmed.

Consider a laminated composite beam with unidirectional plies at 70 degrees, length of $0.5 \mathrm{~m}$, width of $0.1 \mathrm{~m}$, and thickness of $5 \mathrm{~mm}$, with a crack introduced at midspan. The fiber has an elastic modulus of 275.6 GPa, shear modulus of $114.8 \mathrm{GPa}$, Poisson's ratio of 0.2 , and a density of $1900 \mathrm{~kg} / \mathrm{m}^{3}$. The matrix has an elastic modulus of $2.76 \mathrm{GPa}$, shear modulus of $1.036 \mathrm{GPa}$, and Poisson's ratio of 0.33 with a density of $1600 \mathrm{~kg} / \mathrm{m}^{3}$. Moreover, the laminate is designed with a fiber volume fraction of 0.5 .

Natural frequencies of this beam are presented in Table 1 for various crack sizes. For a beam with no damage, the natural frequencies calculated using both the DFE and DSM are nearly identical. When a crack is introduced, and the size of the crack is increased, a reduction in the fundamental frequencies is observed for all modes. The amount of frequency decay depends on the type of mode and stress intensity factor most influencing the crack mode. For example, the first three modes are presented in Figures 5-7 for both bending and twisting displacements. The mode most influenced by the introduction of a crack, both by change in frequency value and modal displacement, is the third mode, which is prominently influenced by torsion.

4.2. Multiple Crack Detection. Consider a slender beam assembled with DFE beam elements with DFCE elements at 
TABLE 1: Natural frequencies of a composite beam with a crack at midspan.

\begin{tabular}{lccc}
\hline Crack ratio & 1st mode $(\mathrm{Hz})$ & 2nd mode $(\mathrm{Hz})$ & 3rd mode $(\mathrm{Hz})$ \\
\hline No crack, DSM & 11.97 & 70.92 & 145.80 \\
No crack, DFE & 11.97 & 71.08 & 145.85 \\
$\alpha=0.2$ & 10.92 & 70.35 & 119.85 \\
$\alpha=0.4$ & 7.78 & 67.53 & 90.33 \\
$\alpha=0.6$ & 4.16 & 62.29 & 79.64 \\
\hline
\end{tabular}

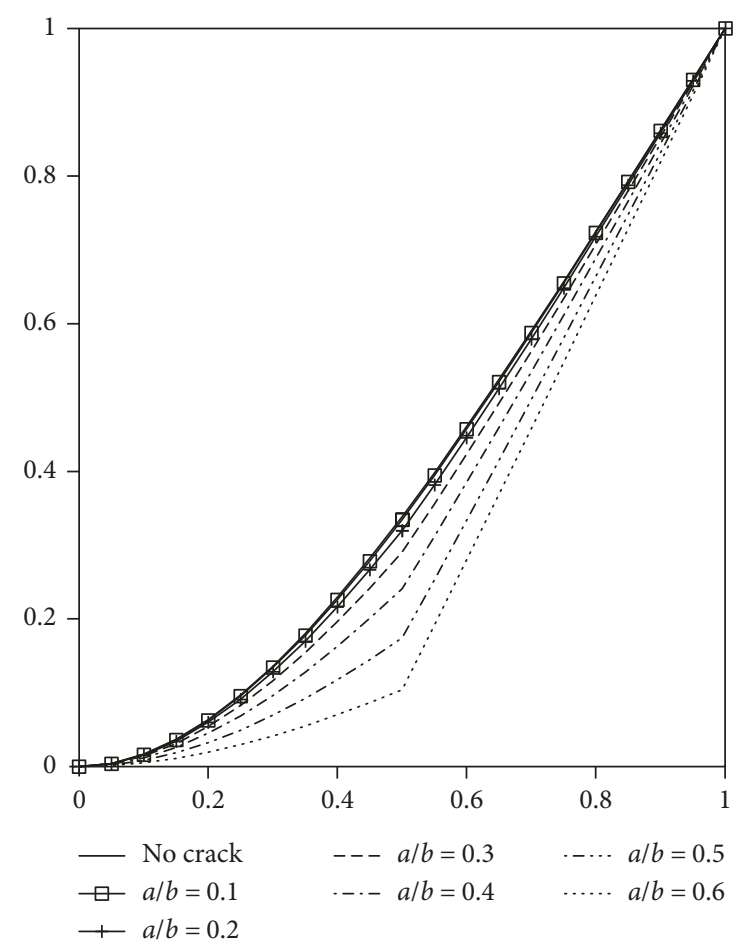

(a)

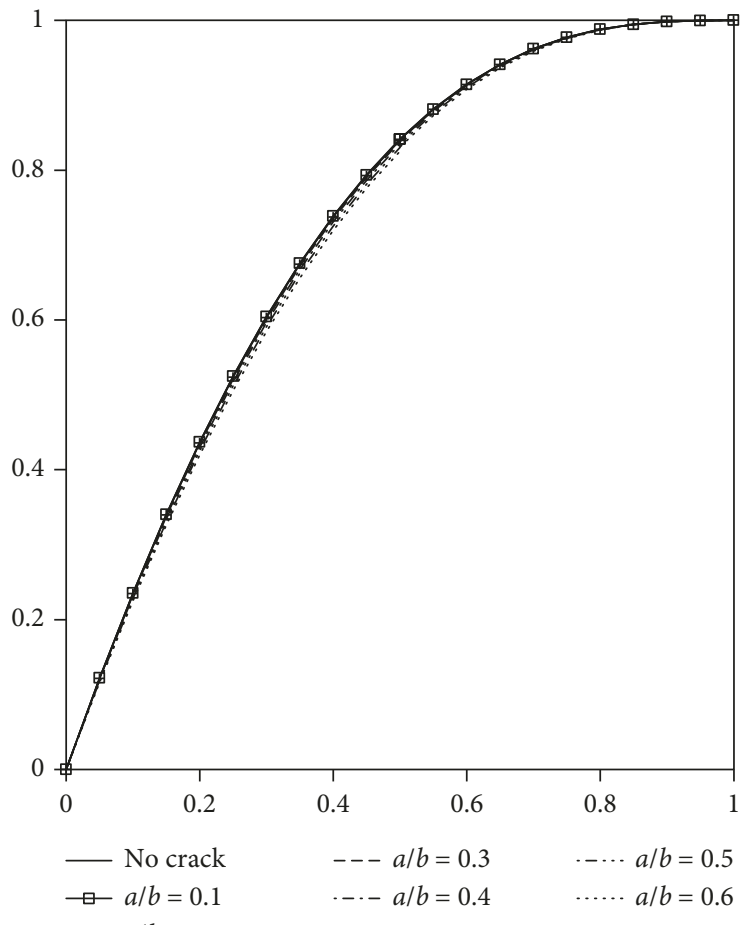

(b)

FIGURE 5: First mode: (a) bending and (b) torsion of a composite beam with a crack located at $50 \%$ span and unidirectional ply angle of $70^{\circ}$.

the appropriate damaged locations. The beam is now considered to be damaged in two distinct locations by two through thickness edge cracks present at $\eta_{1}=0.1$ and $\eta_{2}=$ 0.8 with corresponding crack sizes of $\alpha_{1}=0.3$ and $\alpha_{2}=0.6$. Mechanical and geometric properties of this beam remain identical as the previous example with exception of the unidirectional ply angle of 15 degrees. For expediency, a cracked beam numbering system is implemented such that a multicracked beam with the first crack $\eta_{1}, \alpha_{1}$ and the second crack $\eta_{2}, \alpha_{2}$ is referred to as a $\left[\begin{array}{lllll}10 \eta_{1} & 10 \alpha_{1} & 10 \eta_{2} & 10 \alpha_{2}\end{array}\right]$ beam. For example, the current beam is a [1386] beam. An illustration of this nondimensional multicracked beam is shown in Figure 8.

The motivation for presenting this example is to show that a multicracked detection algorithm can be used to successfully converge on more than one crack, for both size and location, using frequency data. The first five natural frequencies are calculated and presented in Table 2, for both a damaged and intact laminated composite beam. Primary and secondary damage indexes are then calculated using the method described in Section 3 (Figures 9 and 10). These results are based on zero error between experimentally collected frequencies and frequencies calculated using DFCE.

It is reasonable to conclude, natural frequencies collected by experiment have uncertainty in measurement. Subsequently, it is practical to test the detection methodology with error included in these pseudoexperimental frequency values, in the order of $2-3 \%$. Consequently, this structural health monitoring technique requires additional modes to accurately converge on the location and size of each crack, namely, 5 modes of free vibration (Figures 11 and 12). In contrast, detection of cracks when zero error exists in measured frequencies ideally requires the minimum number of modes ( 2 modes). It is important to note the order of the frequencies when forming the damage indices. Natural frequencies must be ordered based on the largest to smallest percent difference between intact and damaged frequencies. This is specific requirement when searching for damage in laminated composite beams [4]. 


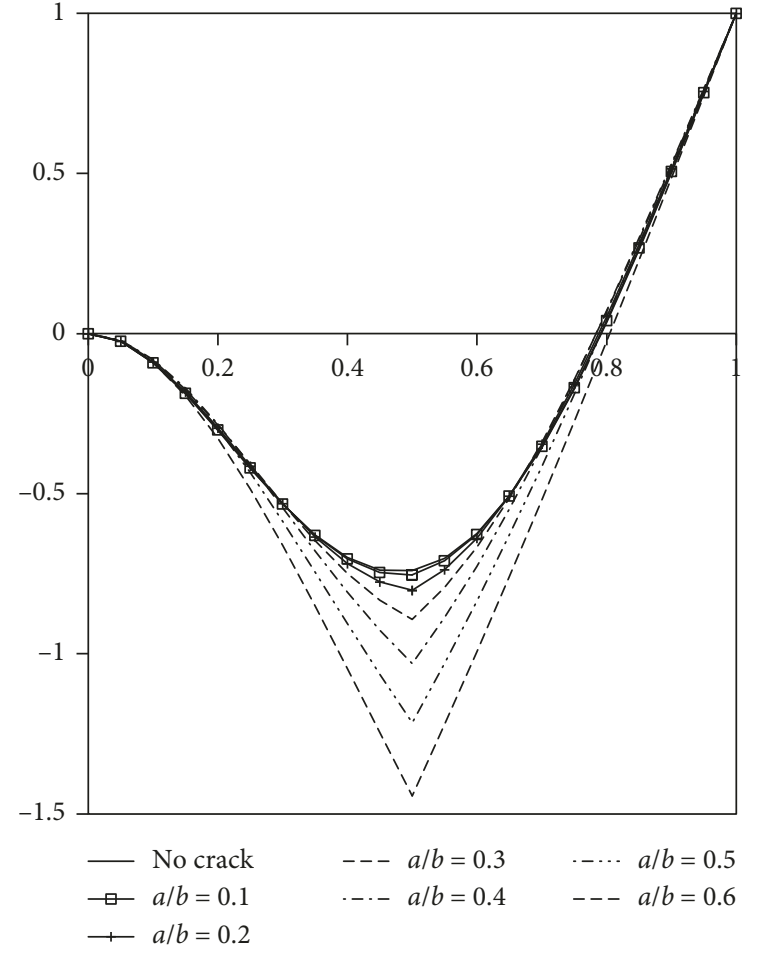

(a)

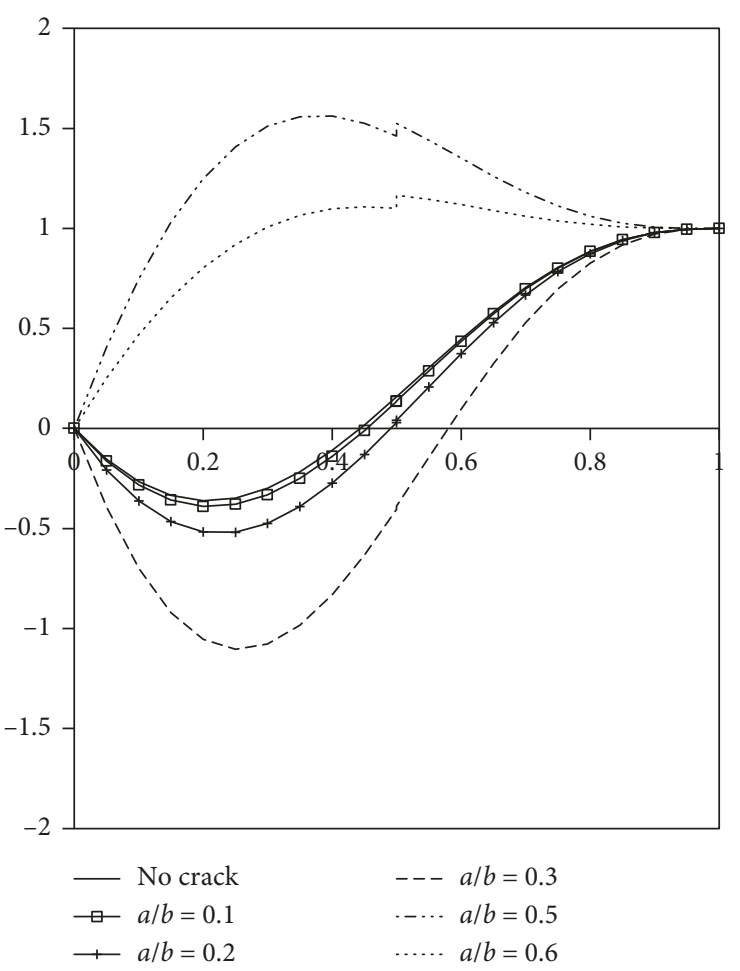

(b)

FIGURE 6: Second mode: (a) bending and (b) torsion of a composite beam with a crack located at $50 \%$ span and unidirectional ply angle of $70^{\circ}$.

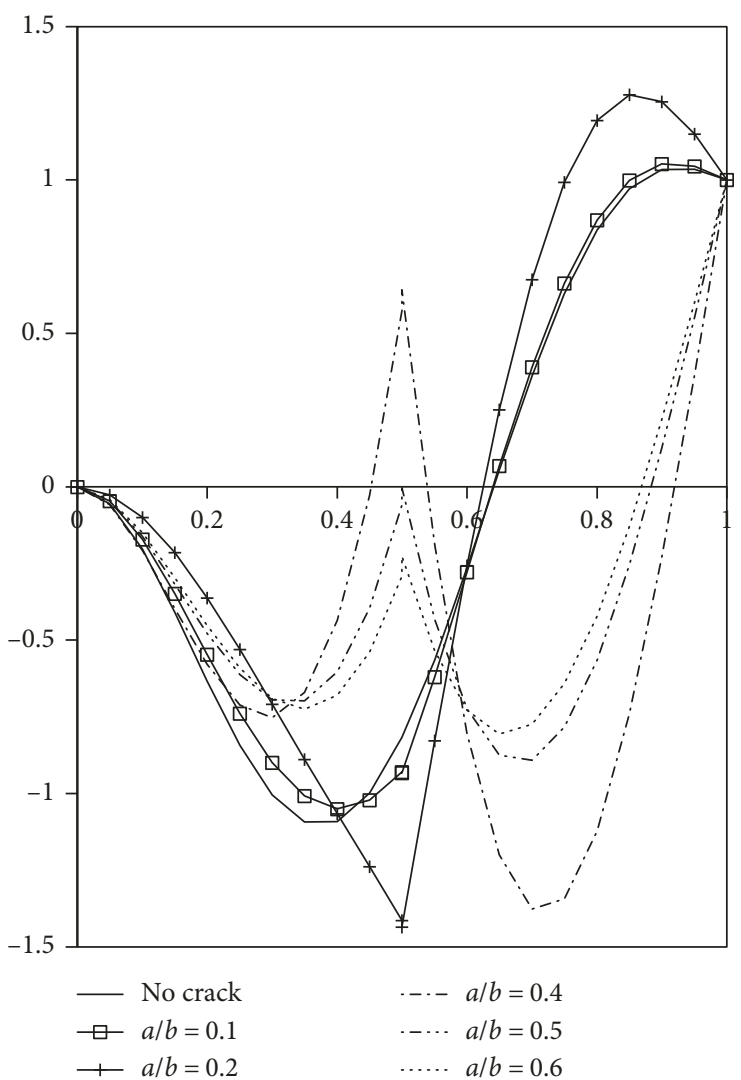

(a)

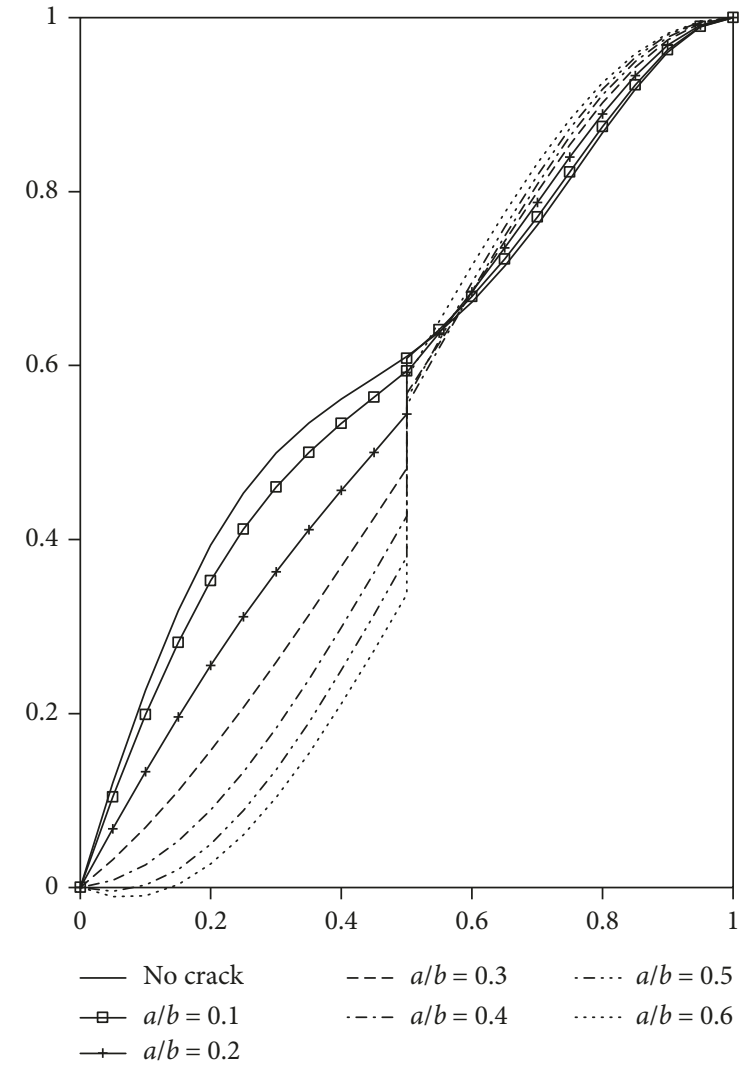

(b)

Figure 7: Third mode: (a) bending and (b) torsion of a composite beam with a crack located at $50 \%$ span and unidirectional ply angle of $70^{\circ}$. 
TABLE 2: Frequency data collected for an intact and multicracked Euler-Bernoulli [1386] laminated composite beam.

\begin{tabular}{lccccc}
\hline & Mode 1 & Mode 2 & Mode 3 & Mode 4 & Mode 5 \\
\hline$f_{\mathrm{i}}(\mathrm{Hz})$ & 6.82 & 42.72 & 71.40 & 119.59 & 214.22 \\
$f_{\mathrm{d}}(\mathrm{Hz})$ & 5.65 & 29.88 & 67.62 & 69.87 & 176.40 \\
Measured error $(\%)$ & 2.8 & 2.8 & 3 & 2 & 2.3 \\
$\widetilde{f}_{\mathrm{d}}$ with error $(\mathrm{Hz})$ & 5.81 & 30.72 & 69.65 & 71.27 & 180.46 \\
\hline
\end{tabular}

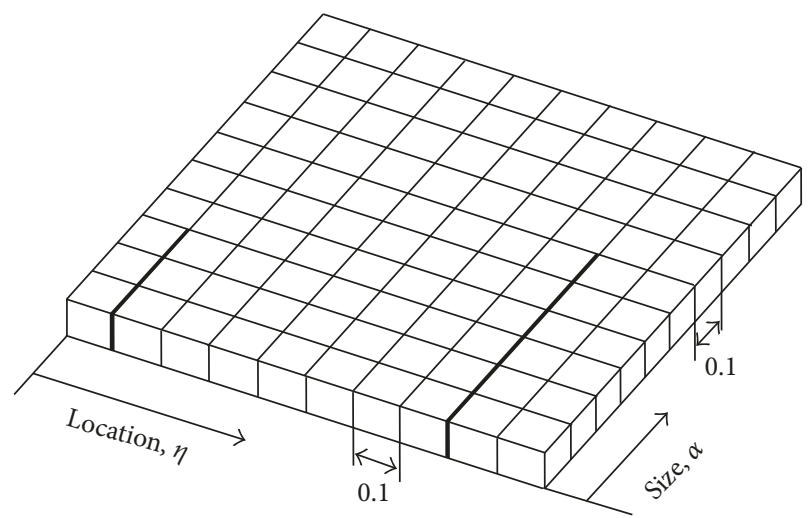

Figure 8: Nondimensional plot $(\eta=y / L, \alpha=a / b)$ with actual crack locations and sizes illustrated for an Euler-Bernoulli and St. Venant torsion [1386] beam.

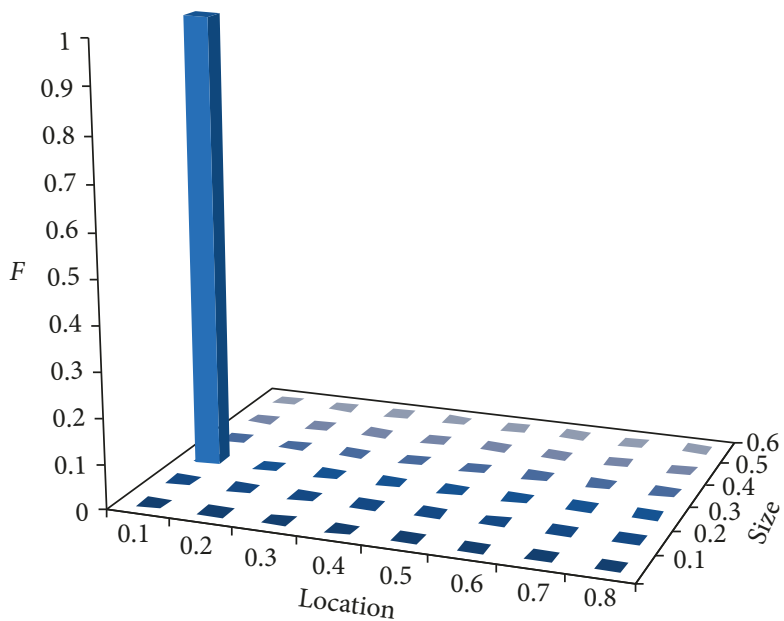

FIgURE 9: Damage index for the first crack of a multicracked [1386] composite beam with no measured errors.

\section{Conclusion}

This paper presents a robust method for detecting multiple cracks in laminated composite bending-torsion coupled cantilevered beams using frequency data, a type of nondestructive testing (NDT). In addition, a dynamic finite cracked element (DFCE) is formulated where the trigonometric shape functions used to generate the stiffness matrix are fundamentally frequency dependent. It has been shown, by applying DFCEs with a dual damage index methodology, damages in the form of through thickness edge cracks can be detected for both size and location. The results have further demonstrated that cracks are identified when error in

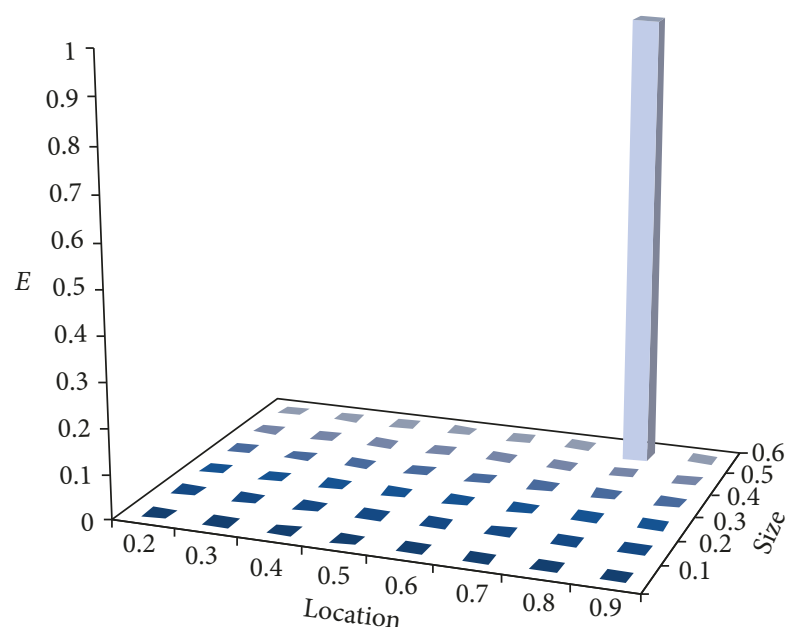

FIGURE 10: Damage index for the second crack of a multicracked [1386] composite beam with no measured errors.

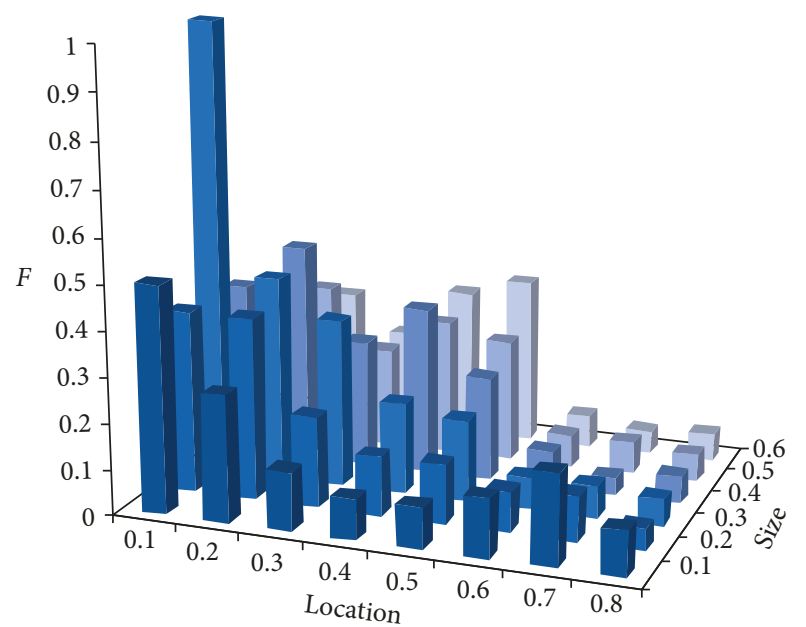

Figure 11: Damage index for the first crack of a multicracked [1386] composite beam using 5 modes with measured error ranging from 2 to $3 \%$.

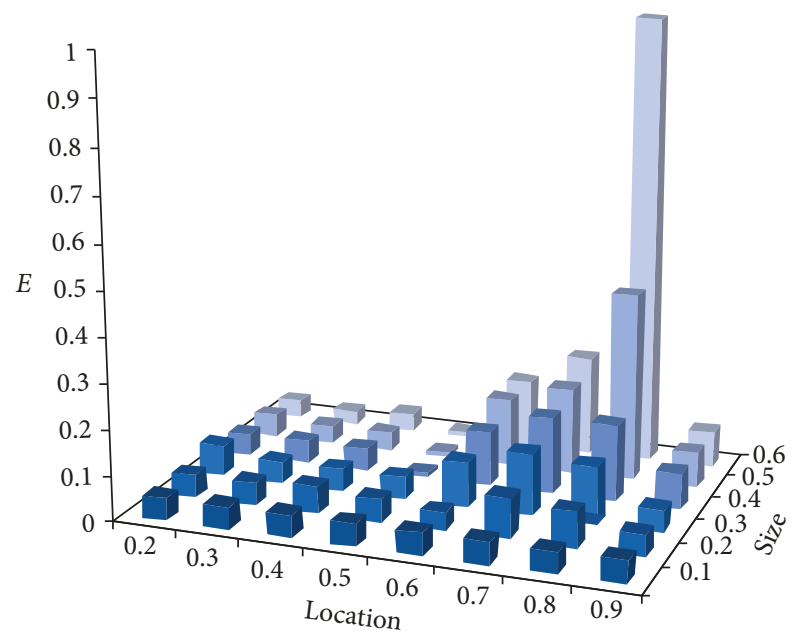

FIGURE 12: Damage index for the second crack of a multicracked [1386] composite beam using 5 modes with measured error ranging from 2 to $3 \%$. 
measured frequencies exists, by using multiple modes free vibration.

\section{Appendix}

\section{$\mathbf{A}$}

The following relationships define the effective rigidities of a unidirectional laminate beam $[12,13]$.

Flexural rigidity:

$$
\mathrm{EI}=b\left(\frac{D_{22}-D_{12}^{2}}{D_{11}}\right) .
$$

Torsional rigidity:

$$
\mathrm{GJ}=4 b\left(\frac{D_{66}-D_{16}^{2}}{D_{11}}\right) .
$$

Coupled bending-torsion rigidity:

$$
K=2 b\left(\frac{D_{26}-D_{12} D_{16}}{D_{11}}\right),
$$

where the bending terms of the constitutive equation are

$$
D_{i j}=\frac{1}{3} \sum_{k=1}^{\mathrm{NL}}\left(\bar{Q}_{i j}\right)_{k}\left(h_{k}^{3}-h_{k-1}^{3}\right) \text {, }
$$

where $\bar{Q}_{i j}$ are the reduced stiffness coefficients of a unidirectional laminate.

B

Four dynamic trigonometric shape functions (DTFSs) pertaining to bending are

$$
\begin{aligned}
& N_{\mathrm{f} 1}=\frac{\{\cos (\beta(1-\xi)) \cosh \beta-\cos (\beta \xi)+\cosh (\beta((1-\xi)) \cos \beta-\cosh (\beta \xi)+\sinh (\beta((1-\xi)) \sin \beta-\sin (\beta((1-\xi)) \sinh \beta\}}{\Delta}, \\
& N_{\mathrm{f} 2}=\frac{(1 / \beta)\{-\sin (\beta((1-\xi)) \cosh \beta-\sin (\beta \xi)+\cosh (\beta(1-\xi)) \sin \beta-\sinh (\beta(1-\xi)) \cos \beta+\cos (\beta(1-\xi)) \sinh \beta-\sinh (\beta \xi)\}}{\Delta}, \\
& N_{\mathrm{f} 3}=\frac{\{-\cosh (\beta(1-\xi))+\cosh (\beta \xi) \cos \beta+\cos (\beta \xi) \cosh \beta-\cos (\beta(1-\xi))+\sinh (\beta \xi) \sin \beta-\sin (\beta \xi) \sinh \beta\}}{\Delta}, \\
& N_{\mathrm{f} 4}=\frac{(1 / \beta)\{\sinh (\beta(1-\xi))-\cosh (\beta \xi) \sin \beta-\cos (\beta \xi) \sinh \beta+\sin (\beta(1-\xi))+\sinh (\beta \xi) \cos \beta+\sin (\beta \xi) \cosh \beta\}}{\Delta},
\end{aligned}
$$

where

$$
\Delta=2(\cosh \beta \cos \beta-1) .
$$

Similar DTSFs have also been used previously [5, 8]. For torsion, one can write

$$
\begin{aligned}
N_{\mathrm{t} 1} & =\frac{\sin \gamma(1-\xi)}{\sin \gamma}, \\
N_{\mathrm{t} 2} & =\frac{\sin (\gamma \xi)}{\sin \gamma}, \\
\beta & =\sqrt[4]{\frac{m \omega^{2} l_{k}^{4}}{\mathrm{EI}}} \\
\gamma & =\sqrt{\frac{I_{\alpha} \omega^{2} l_{k}^{2}}{\mathrm{GJ}}} .
\end{aligned}
$$

\section{Data Availability}

No data were used to support this study.

\section{Conflicts of Interest}

The authors declare that they have no conflicts of interest.

\section{Acknowledgments}

The authors wish to acknowledge the support provided by the Natural Sciences and Engineering Research Council of Canada (NSERC), Ontario Graduate Scholarship (OGS) Program, and Ryerson University.

\section{References}

[1] P. Cawley and R. D. Adams, "The location of defects in structures from measurements of natural frequencies," Journal of Strain Analysis for Engineering Design, vol. 14, no. 2, pp. 49-57, 1979.

[2] S. W. Doebling, C. R. Farrar, and M. B. Prime, "A summary review of vibration-based damage identification methods," Shock and Vibration Digest, vol. 30, no. 2, pp. 91-105, 1998.

[3] R. D. Adams, P. Cawley, C. J. Pye, and B. J. Stone, "A vibration technique for non-destructively assessing the integrity of structures," Journal of Mechanical Engineering Science, vol. 20, no. 2, pp. 93-100, 1978.

[4] K. Wang, Vibration analysis of cracked composite bendingtorsion beams for damage diagnosis, Ph.D. thesis, Faculty of the Virginia Polytechnic Institute and State University, Blacksburg, VA, USA, 2004.

[5] S. Jayasinghe and S. M. Hashemi, "Frequency dependant trigonometric shape functions for thin rectangular plate 
elements," in Proceedings of 23rd International Congress on Sound Vibration (ICSV'23), Athens, Greece, July 2016.

[6] J. R. Banerjee and F. W. Williams, "Free vibration of composite beams-an exact method using symbolic computation," Journal of Aircraft, vol. 32, no. 3, pp. 636-642, 1995.

[7] W. H. Wittrick and F. W. Williams, "A general algorithm for computing the natural frequencies of elastic structures," Quarterly Journal of Mechanics and Applied Mathematics, vol. 24, no. 3, pp. 263-284, 1971.

[8] S. M. Hashemi and S. Borneman, "Doubly-coupled vibrations of nonuniform composite wings: a dynamic finite element," in Mathematical Problems in Engineering, Aerospace and Sciences, S. Sivasundaram, Ed., vol. 5, pp. 141-152, Cambridge Scientific Publishers, 2011, ISBN 978-1-904868-79-8.

[9] K. Nikpour and A. D. Dimarogonas, "Local compliance of composite cracked bodies," Composites Science and Technology, vol. 32, no. 3, pp. 209-223, 1988.

[10] H. Tada, P. C. Paris, and G. R. Irwin, The Stress Analysis of Cracks Handbook, ASME Press, 3rd edition, 2000.

[11] G. Bao, S. Ho, and Z. Suo, "The role of material orthotropy in fracture specimens for composites," International Journal of Solids and Structures, vol. 29, no. 9, pp. 1105-1116, 1992.

[12] J. M. Berthelot, Composite Materials Mechanical Behaviour and Structural Analysis, Springer-Verlag, New York, NY, USA, 1999.

[13] R. M. Jones, Mechanics of Composite Materials, Taylor and Francis Inc., Oxfordshire, UK, 2nd edition, 1998. 


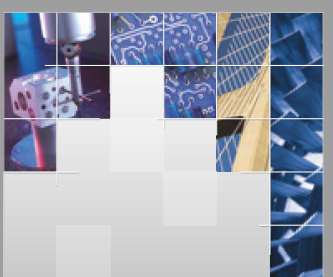

\section{Enfincering}
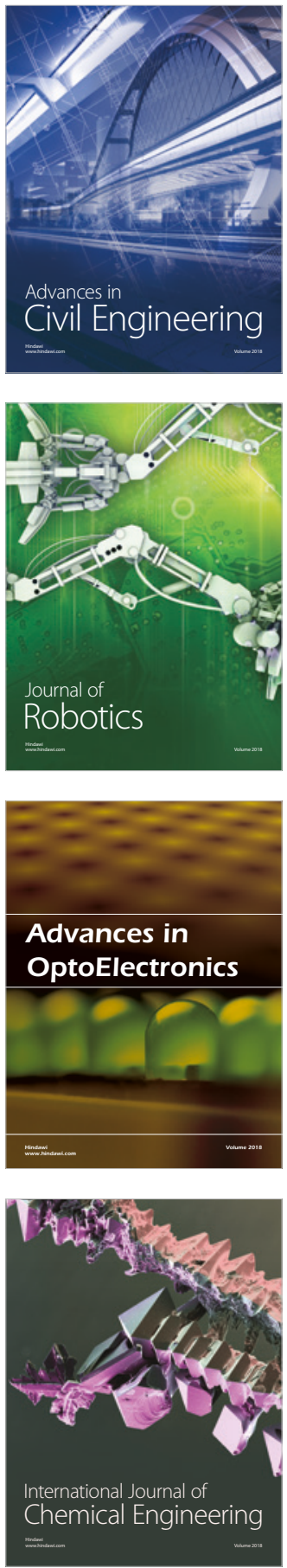

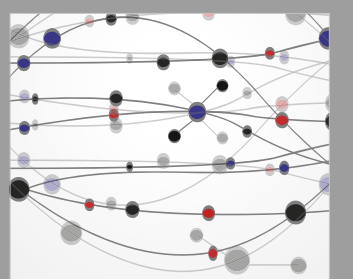

\section{Rotating \\ Machinery}

The Scientific World Journal

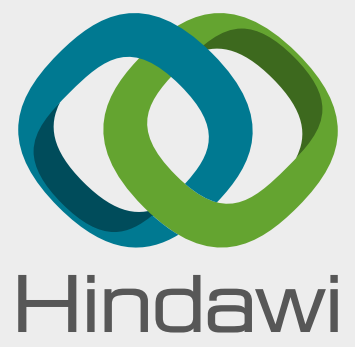

Submit your manuscripts at

www.hindawi.com
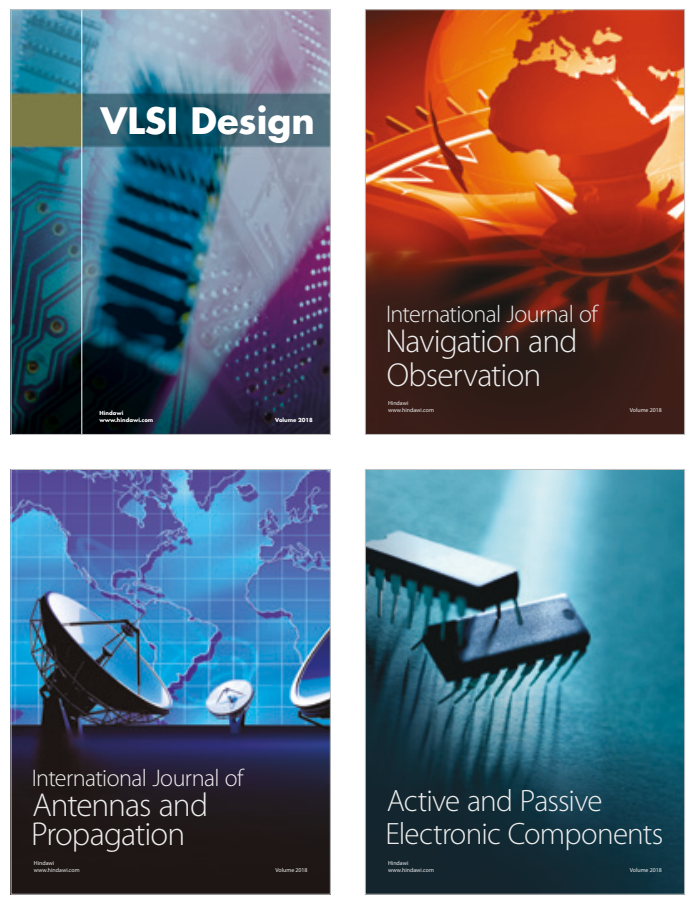
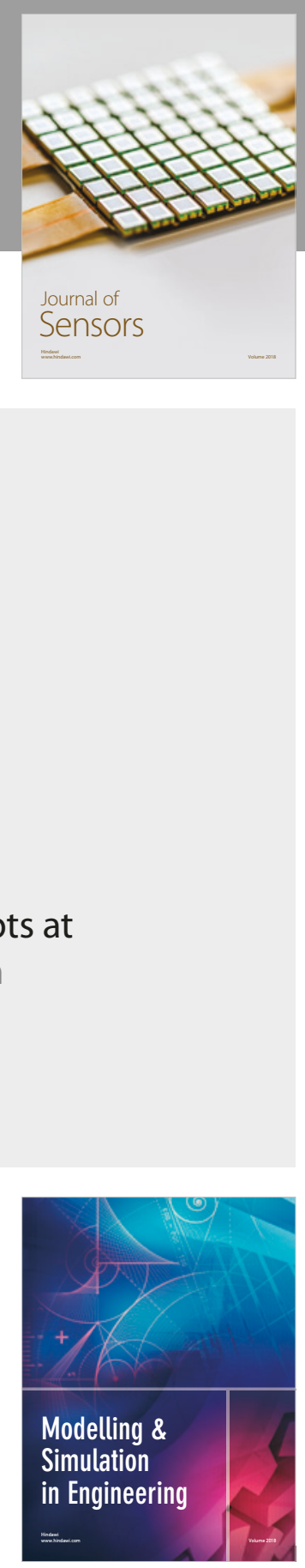

\section{Advances \\ Multimedia}
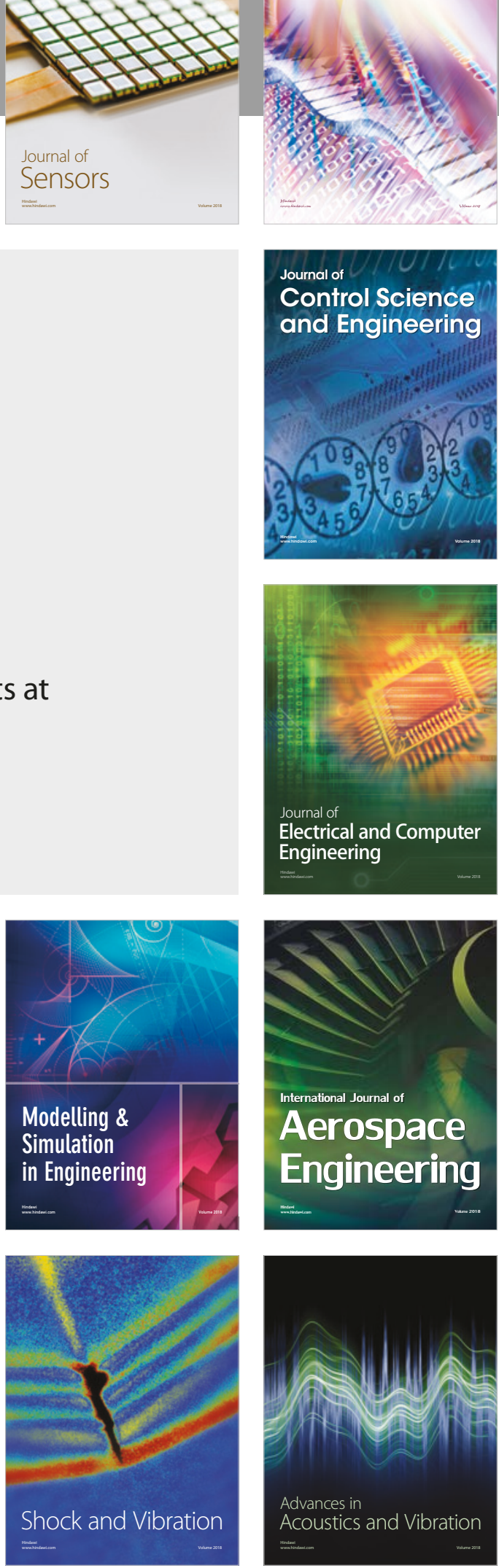